\title{
Results of the first coincident observations by two laser-interferometric gravitational wave detectors
}

\author{
D. Nicholson ${ }^{\text {a }}$, C.A. Dickson ${ }^{\text {a }}$, W.J. Watkins ${ }^{\text {a }}$, B.F. Schutz ${ }^{\text {a }}$, J. Shuttleworth ${ }^{\text {a }}$, G.S. Jones ${ }^{\text {a }}$, \\ D.I. Robertson $^{b}$, N.L. Mackenzie ${ }^{b}$, K.A. Strain ${ }^{b}$, B.J. Meers ${ }^{b}$, G.P. Newton ${ }^{b}$, H. Ward ${ }^{b}$, \\ C.A. Cantley ${ }^{b}$, N.A. Robertson ${ }^{b}$, J. Hough ${ }^{b}$, K. Danzmann ${ }^{c}$, T.M. Niebauer ${ }^{c}$, A. Rüdiger ${ }^{c}$, \\ R. Schilling ${ }^{c}$, L. Schnupp ${ }^{c}$, W. Winkler ${ }^{c}$ \\ a Department of Physics and Astronomy, University of Wales College of Cardiff, Cardiff, UK \\ b Department of Physics and Astronomy, University of Glasgow, Glasgow, UK \\ c Max Planck Institute for Quantum Optics, Garching, Germany
}

Received 10 April 1996; accepted for publication 16 May 1996

Communicated by P.R. Holland

\begin{abstract}
We report an upper bound on the strain amplitude of gravitational wave bursts in a waveband from around $800 \mathrm{~Hz}$ to $1.25 \mathrm{kHz}$. In an effective coincident observing period of 62 hours, the prototype laser interferometric gravitational wave detectors of the University of Glasgow and Max Planck Institute for Quantum Optics, have set a limit of $4.9 \times 10^{-16}$, averaging over wave polarizations and incident directions. This is roughly a factor of 2 worse than the theoretical best limit that the detectors could have set, the excess being due to unmodelled non-Gaussian noise. The experiment has demonstrated the viability of the kind of observations planned for the large-scale interferometers that should be on-line in a few years time.
\end{abstract}

\section{Introduction}

Gravitational radiation is expected from a wide range of astrophysical sources such as stellar collapses, mergers of neutron star and black hole binaries, pulsars, and from the very early universe. In order to have an appreciable chance of detecting this radiation, theoretical calculations indicate that gravitational wave detectors should attain an effective strain sensitivity $h$ better that about $10^{-21}$ over a bandwidth from a few hundred $\mathrm{Hz}$ to about $1 \mathrm{kHz}$ $[1,2]$. It is anticipated that this target will be reached in the next few years by large-scale laser interferometric detectors [3-5]. A comprehensive overview of gravitational wave detection can be found in two recent books $[6,7]$.

At 15:02 (GMT) on 02 March 1989 two prototype gravitational wave detectors, one operated by the University of Glasgow (UG) and the other by the Max Planck Institute for Quantum Optics (MPQ), participated in a joint observing run over a period of 100 hours. The motivations for this run were twofold. First, to demonstrate the practicality of making long-term coincident observations with interferometers, and second to provide real data with all its inherent complexities for testing out a range of signal analysis programs. This was the first time that two interferometers had been run in coincidence for such a length of time. The 
noise performances of the detectors during the run were poorer by more than a factor of ten times what the prototypes could achieve today. They might have been able to detect a nearby $(1 \mathrm{kpc})$ gravitational collapse event in our Galaxy, the probability of which in any 100-hour period may be between $10^{-5}$ and $10^{-6}$. We give brief descriptions of the detectors, the experiment, and the results in this paper. Further details are given elsewhere $[8,9]$.

\section{The detectors}

The UG detector is a Fabry-Perot interferometer situated at latitude $55.86^{\circ} \mathrm{N}$, longitude $4.23^{\circ} \mathrm{W}$; its arms are orthogonal, at $193^{\circ}$ and $283^{\circ}$ clockwise from north. The MPQ detector is a Michelson delay-line interferometer located at $48.24^{\circ} \mathrm{N}, 11.68^{\circ} \mathrm{E}$; its arms are rotated by angles of $31^{\circ}$ and $121^{\circ}$ from north. The separation of the detectors on a chord through the Earth is $1370 \mathrm{~km}$, corresponding to a wave travel time (at the speed of light) of $4.6 \mathrm{~ms}$. A measure of the common sensitivity of the detectors to impinging gravitational waves is provided by the overlap between the detectors' antenna patterns. The prototype detectors have patterns that overlap by $89 \%$ implying that their arms are nearly aligned. As we shall see, this feature simplifies the coincidence analysis.

Fig. 1a displays the linear strain noise spectral density of the UG detector at a time when the detector was functioning normally and close to optimally. Above about $1.5 \mathrm{kHz}$, the instrument operated near to it's shot-noise limited sensitivity. The excess noise below this frequency was due mainly to ground vibrations and mechanical resonances. The $h$ strain sensitivity of the UG detector, in a frequency band $0-1.25 \mathrm{kHz}$ which we motivate later, most often had a measured value of around $2 \times 10^{-17}$. At times, however, the sensitivity was observed to improve to a level $10^{-17}$, and conversely there were transitory spells when the sensitivity would degrade by more than a factor of five times this value.

The linear strain noise spectral density curve for the MPQ detector, again during normal and near-optimal operation, is shown in Fig. 1b. It shows features that are broadly similar to the corresponding curve for the UG detector. High-pass and low-pass (anti-aliasing) filters, with corner frequencies of $320 \mathrm{~Hz}$ and $4 \mathrm{kHz}$ respectively, were applied to the data before it was recorded. Their effects are illustrated by the faint line in the figure. Noise peaks are evident at a frequency near $3.5 \mathrm{kHz}$. Their origin is known to lie in mechanical resonances within the detector. The typical 0-1.25 $\mathrm{kHz} h$ strain sensitivity of the MPQ detector during the experiment had a measured value around $3 \times 10^{-17}$. About midway through the experiment this sensitivity was observed to degrade by more than a factor of two due to loss of alignment of the laser beams in the vacuum tubes. In common with the observed behaviour of the UG sensitivity, there were also rare, transitory, periods when the sensitivity of the MPQ detector was poorer by more than a factor of five times its typical measured value.

\section{The observing run}

In this section we describe briefly the operational details of the experiment. Further information can be found elsewhere $[8,10,9]$.

The UG detector produced $60 \mathrm{kB} / \mathrm{s}$ of data recorded onto 28 tapes by an Exabyte tape drive. One third of this was the main output data stream, called the secondary error point signal. The rest was primarily housekeeping data. The whole set amounted to about $20 \mathrm{~GB}$ of data for the entire experiment.

The MPQ detector produced about $44 \mathrm{kB} / \mathrm{s}$ of data, which were recorded onto 94 standard 6250 Bpi 9track tapes. The main interferometer signal was sampled at $10 \mathrm{kHz}$ and compounded by several housekeeping streams. A total data set of about $15 \mathrm{~GB}$ was amassed during the experiment.

Interferometers are intrinsically more difficult to operate than bar detectors, since they involve a number of active control systems that must constantly be monitored and occasionally corrected. Designs for the large-scale interferometers will incorporate many control features whose function is to minimize the need for operators to intervene with the running of the detectors. The present prototypes do not incorporate such features, since they were designed as scientific development test-beds rather than as observatories. It was therefore very encouraging that the prototypes performed so well: they acquired data simultaneously during $88 \%$ of the experiment, and operated close to their optimum sensitivity simultaneously for $62 \%$ of the experiment [9]. 

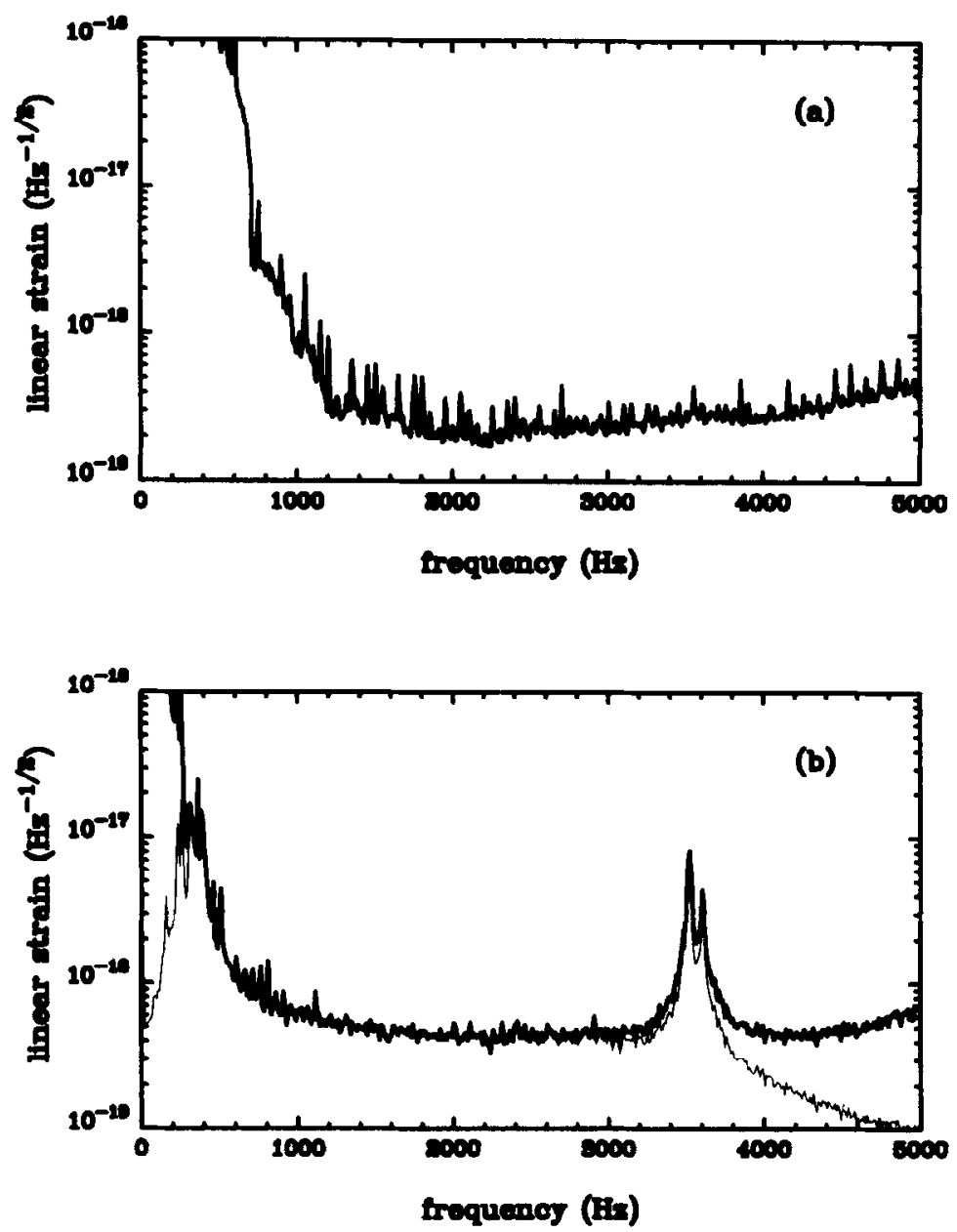

Fig. 1. Linear noise spectral density curves for the (a) University of Glasgow (UG) and (b) Max Planck Institute for Quantum Optics (MPQ) prototype gravitational wave detectors under normal and near-optimal operation during the 100-hour experiment. The faint line in curve (b) illustrates the effect of anti-aliasing and high-pass filters applied to data from the Max Planck detector. which were removed for the data analysis.

\section{The analysis method}

Several of the general issues that arise in the analysis of gravitational wave data have been addressed in recent monographs $[11,15]$. A limited analysis of the UG data has been performed [8], and the MPQ data set has been searched for a pulsar signal [10]. Our focus here is on short-time-scale bursts that could be produced by supernova explosions. Subsequent publications will analyse the same data to place upper limits on the gravitational wave flux of periodic waves impinging from a small solid angle on the sky [12], and of a stochastic background [13].
Our analysis method was basically to compute cross-correlation integrals for each individual data stream in turn, with a filter weighted by the inverse of the power spectral density of the noise for that data stream. This is the classical method of matchedfiltering [16]. The inverse noise spectral density weighting in the filter suppresses contributions to the integral from frequency bands where the data is very noisy. It was important, of course, to remove the antialiasing and high-pass filters from the MPQ data to ensure that this weighting would be done properly.

The Fourier transform of the filter we employed is given simply by $\Pi(f / 2500 \mathrm{~Hz})$ where $\Pi(x)$ is 
the "boxcar function" [17], of unit value for $|x|<$ $1 / 2$ and zero otherwise. The filter's output is thus the inverse Fourier transform of the noise-weighted data stream using only frequencies below the cutoff of 1.25 $\mathrm{kHz}$. It is evident from Fig. 1 that the detectors are most sensitive in a frequency range from about $800 \mathrm{~Hz}$ to $3 \mathrm{kHz}$, and this overlaps with only $1 / 5$ of the bandwidth of our burst filter. However, the performance of resonant bar detectors is generally quoted in terms of their sensitivity to flat spectrum bursts in the approximate frequency range $0-1.5 \mathrm{kHz}$, even though bars are only sensitive in a much narrower band [20]. Our burst filter was therefore chosen for reasons of compatibility with the model adopted by the resonant bar community, in order to facilitate comparisons between respective sets of results. Some numerical simulations have also reinforced the expectation that supernova bursts will be broadband in the kilohertz region [18].

\section{The analysis software}

The 100-hour experiment was undertaken with a view to gaining practical experience under realistic conditions and offering guidance for the development of larger detectors. This motivated our decision to analyze the data with computer programs that could serve as prototypes of programs that will have to process the data of the large interferometers in real time. This software, designed by the Cardiff group, is described in detail in a Ph.D. thesis [14].

\subsection{Identifying coincidences}

The programs produced lists of "events". An event is defined as a set of contiguous data samples where the output of the filter exceeds a predetermined threshold. The threshold was set low enough to generate a reasonable number of events for the statistics of the coincidence search. Several diagnostics for each event were recorded onto Exabyte tapes, including the time, duration, and maximum amplitude of the event, and numbers characterizing the detector, such as the broadband sensitivity to kilohertz bursts in short time slices of filter output and various housekeeping indicators (microphone and seismometer signals etc.) at the time of the event.

A coincident event is defined to be a pair of events from the two data streams that occur within the accep- tance window given by the gravitational wave travel time between the detectors. The list of coincidences inevitably contains some with very large amplitude, and one has to assess their significance. It is important to formulate a priori criteria for accepting events, without reference to the specific properties of the data set. Our analysis proceeded through three levels of vetoes.

At the first level, we looked at housekeeping data and applied vetoes if the detectors were not performing correctly, or if an environmental disturbance had occurred which may have affected the detector. The vetoes were partially, but not entirely, successful in eliminating periods when the detectors were performing below par. There were unvetoed times during the experiment when the kilohertz burst sensitivities of the detectors were five times larger than their typical measured values.

The second level accepted only data from periods where the sensitivity of the two detectors was nearly optimum. At other times, the information from the experiment was less useful. For around $20 \%$ of the time that the UG detector took data, it's kilohertz burst sensitivity exceeded $4 \times 10^{-17}$. Similarly, for $20 \%$ of it's observing time the MPQ detector had a kilohertz burst sensitivity in excess of $5 \times 10^{-17}$. A decision was made to reject coincidences if the events from the individual streams belonged to times when the filtered strain noise fell into the respective $20 \%$ tails. This reduced the effective coincidence observing period of the detectors to around 62 hours.

At the third level, events that survived the first two vetoes were tested against a simple model: if they were genuine gravitational waves, they ought to have shown the same intrinsic strain amplitude in both detectors, apart from the effects of detcctor noise. This is by virtue of the near-alignment of the interferometers' arms which was noted above. We calculated the probability that the two observed strain amplitudes could have been produced by an unknown signal plus independent noise, given the noise level in each detector at the time of the coincidence, and based on a model of independent Gaussian noise in the two detectors. If this probability was less than $0.1 \%$, we rejected the coincidence. The reason for rejecting such events is our assumption that any real gravitational wave events of this strength will be very rare, and it is therefore unreasonable to conclude that a coincidence is caused by a gravitational wave if to do so requires us to assume that, in addition, the detectors were behaving in 
a very unusual way as well when that rare event occurred. We call this the $h$-veto.

Coincidences that survive the three levels of rejection set the upper limit on the sensitivity of our experiment.

\section{Results}

We chose a threshold of $4 \sigma$ for generating events from the filters. The top panel of Fig. 2 shows the signal-to-noise ratio (SNR) distribution of coincident events that pass the housekeeping vetoes during good data periods (levels 1 and 2). A calculation based on the empirical SNR distributions of all the events in each detector that survive these vetoes, yields a probability for the least likely coincident event in this diagram of 0.51 , so none of our events are statistically significant outliers. The bottom panel of the same figure shows events that pass the $h$-veto, so that they could have been generated by a gravitational wave with a reasonable amount of noise on top. The axes here are in strain rather than SNR. The inferred intrinsic amplitude of coincident bursts is the average value of the strains on the two axes. We conclude that our limit on kilohertz bursts of gravitational radiation is $2.2 \times 10^{-16}$. This is the limit if the waves arrive from the zenith with the optimum polarization. The corresponding limit on bursts of any wave polarization coming from any direction on the sky is a factor of $\sqrt{5}$ worse, or $4.9 \times 10^{-16}$.

\section{Discussion}

Our limits are the first obtained over a broad gravitational wave bandwidth. The false-alarm threshold for a single alarm during the effective coincidence observing period, taking into account the light travel-time between the detectors, and assuming a background of independent Gaussian noise in the detectors, is $4.5 \sigma$. Given the typical kilohertz burst sensitivity of the detectors, we estimate that our upper bound on $h$ is only about a factor of roughly 2 worse than the theoretical best limit that these detectors could have set. The most recent result that has been published in any detail for coincident experiments between resonant bars, has set an upper limit on the amplitude of kilohertz bursts of $1 \times 10^{-17}[20]$. This is for a narrow wave-
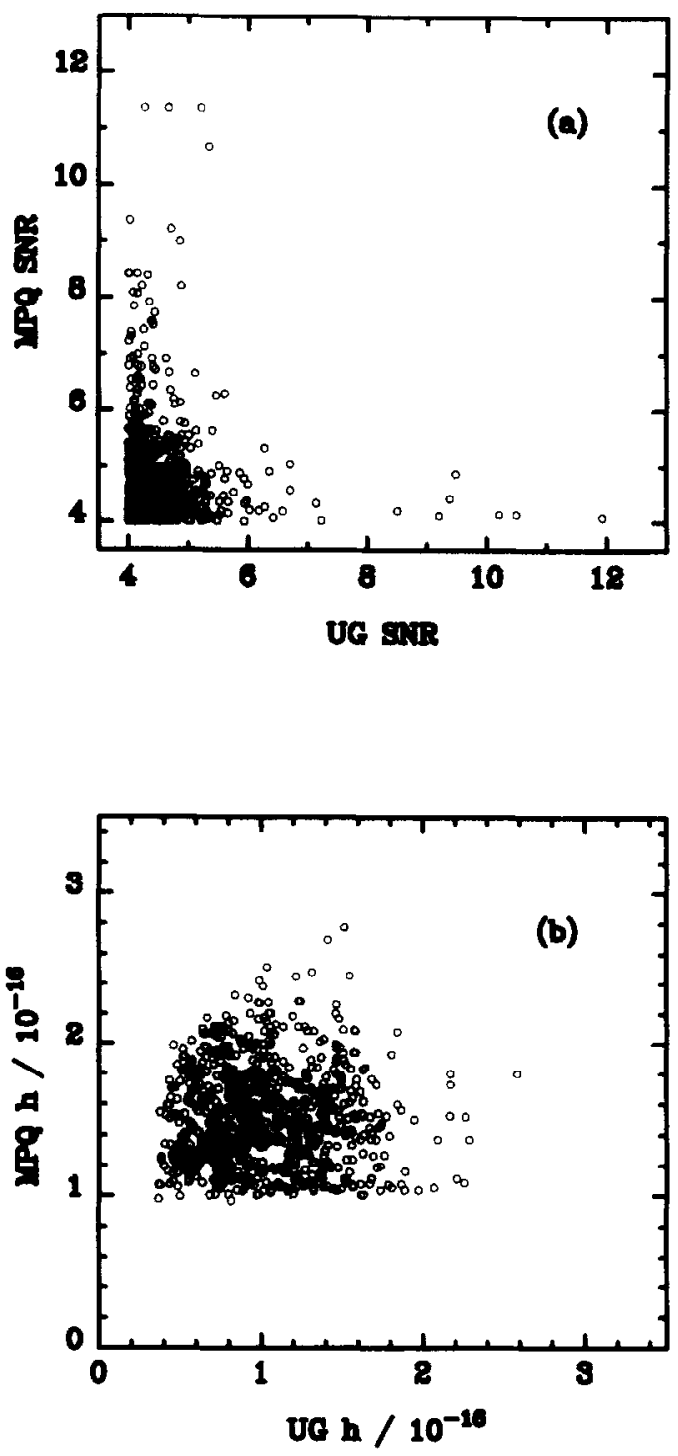

Fig. 2. A signal-to-noise ratio scatter plot (a) of coincident events passing the first two levels of analysis and a strain amplitude scatter plot (b) for events that also pass the $h$-veto. See text for details.

band however (and it is not stated whether this limit incorporates the sky-averaging factor). Interferometer prototypes have been markedly improved since 1989, and would probably come very close to this limit if a similar experiment to the 100 -hour run were performed today. However, the real value of our results is a test of interferometric observing. Our results are very encouraging for large-scale interferometers, since they indicate that attention to detector control and non- 
Gaussian noise could raise the sensitivity and duty cycle of working detectors very close to their optimum performance.

\section{References}

[1] K.S. Thorne, in: 300 years of gravitation, eds. S.W. Hawking and W. Israel (Cambridge Univ. Press, Cambridge, 1987).

[2] B.F. Schutz, Ann. N.Y. Acad. Sci. 571 (1989) 27.

[3] A. Abramovici et al., Science 256 (1992) 325.

[4] C. Bradaschia et al., Nucl. Instrum. Meth. A 289 (1990) 518.

[5] J. Hough et al., Proposal for a $600 \mathrm{~m}$ laser interferometric gravitational wave detector (1994).

[6] D.G. Blair, ed., The detection of gravitational waves (Cambridge Univ. Press, Cambridge, 1991).

[7] P.R. Saulson, Fundamentals of interferometric gravitational wave detectors (World Scientific, Singapore, 1994).

[8] D.I. Robertson, Ph.D. thesis, University of Glasgow (1991).
[9] D. Nicholson et al., in preparation.

[10] T.M. Niebauer, A. Rüdiger, R. Schilling, L. Schnupp, W. Winkler and K. Danzmann, Phys. Rev. D 47 (1992) 3106.

[11] B.F. Schutz, ed., Gravitational wave data analysis (Kluwer, Dordrecht, 1989).

[12] G.S. Jones, Ph.D thesis, University of Wales College of Cardiff (1995).

[13] K.A. Compton, Ph.D thesis, University of Wales College of Cardiff (1996).

[14] W.J. Watkins, Ph.D. thesis, University of Wales College of Cardiff (1991).

[15] B.F. Schutz, in: The detection of gravitational waves, ed. D.G. Blair (Cambridge Univ. Press, Cambridge, 1991).

[16] L.A. Wainstein and V.D. Zubakov, Extraction of signals from noise (Dover, New York, 1962).

[17] R.N. Bracewell, The Fourier transform and its applications, 2nd Ed. (McGraw-Hill, New York, 1978).

[18] L.S. Finn, Ann. N.Y. Acad. Sci. 631 (1991) 156.

[19] C.A. Dickson, Ph.D. thesis, University of Wales College of Cardiff (1993).

[20] E.Amaldi et al., Astron. Astrophys. 216 (1989) 325. 